Bruno Ramalho de Carvalho'

Rosana Maria dos Reis ${ }^{2}$

JOAQUIM COUTINHO NETTO ${ }^{3}$

Marcos Dias de Moura ${ }^{2}$

ANTÔNIO Alberto NOGUERA ${ }^{4}$

RuI Alberto Ferrian ${ }^{5}$

\title{
Molde de látex natural (Hevea brasiliensis) para neovaginoplastia
}

\author{
Natural latex (Hevea brasiliensis) mold for neovaginoplasty
}

Artigos originais

Palavras-chave

Vagina/anormalidades Vagina/cirurgia Procedimentos cirúrgicos em Ginecologia/métodos

Látex

Keywords

Vagina/abnormalities Vagina/surgery Gynecologic surgical procedures/methods Latex

\section{Resumo}

OBJETIVO: avaliar a utilização do molde de látex natural (Hevea brasiliensis) como modificação à neovaginoplastia de Mclndoe e Bannister em pacientes portadoras da síndrome de Mayer-Rokitansky-Küster-Hauser (MRKH). MÉTODOS: análise retrospectiva de nove pacientes com o diagnóstico de síndrome de $M R K H$, submetidas à neovaginoplastia pela técnica de Mclndoe e Bannister com molde de látex natural. Foram avaliadas epitelização, amplitude e profundidade das neovaginas, ocorrência de coitos bem como satisfação e complicações cirúrgicas. RESULTADOS: após cinco semanas do procedimento, oito pacientes apresentavam neovaginas epitelizadas, com profundidade de 7 a $12 \mathrm{~cm}$. Houve um caso de estenose neovaginal completa em decorrência do uso incorreto do molde pela paciente no pós-operatório. Após seguimento mínimo de um ano, todas as pacientes mantinham neovaginas com profundidade de 4 a $8 \mathrm{~cm}$ e capacidade para o coito, com 66,7\% de satisfação. Uma paciente apresentou fístula retovaginal precoce e episódios tardios de fistulização uretrovaginal. Duas pacientes apresentaram estenose distal das neovaginas a longo prazo. Uma delas e a paciente com fístulas foram submetidas a novo procedimento. CONCLUSÕES: o uso do molde de látex natural como modificação à técnica clássica de neovaginoplastia permitiu a criação de neovaginas morfológica e funcionalmente similares ao órgão normal em pacientes com estenose vaginal.

\section{Abstract}

PURPOSE: to evaluate the use of natural latex mold (Hevea brasiliensis) as a modification of Mclndoe and Bannister neovaginoplasty in patients presenting Mayer-Rokitansky-Küster-Hauser (MKRH) syndrome. METHODS: we retrospectively included nine patients presenting MKRH syndrome, who had been submitted to McIndoe and Bannister neovaginoplasty modified by the use of natural latex mold. Neovaginal epithelization and depth, coitus occurrence and satisfaction, and surgical complications were evaluated. RESULTS: five weeks after the procedure, eight patients presented an epithelized 7 to $12 \mathrm{~cm}$ deep neovagina. There was one case of complete neovaginal stenosis, because of incorrect use of the mold. After at least one year, the others maintained 4 to $8 \mathrm{~cm}$ deep neovaginas and capacity for intercourse, with $66.7 \%$ satisfaction. One woman presented precocious rectovaginal fistula and late episodes of uretrovaginal fistulae. Two patients presented distal neovaginal stenosis in long-term follow-up. One of these and the patient with fistulae were submitted to a new procedure. CONCLUSIONS: the use of natural latex mold as a modification of classic neovaginoplasty technique allows the creation of neovaginas morphologically and functionally similar to the normal vagina in patients with vaginal agenesis
Correspondência:

Rosana Maria dos Reis Laboratório de Ginecologia e Obstetrícia - $1^{\circ}$ andar Hospital das Clínicas da Faculdade de Medicina de Ribeirão Preto Avenida Bandeirantes, 3.900 Campus da Universidade de São Paulo - Monte Alegre CEP 14048-900 - Ribeir̃̃o Preto/SP

Fone: (16) 3602-2926 E-mail: romareis@fmrp.usp.br

Recebido

06/11/2007
Departamento de Ginecologia e Obstetrícia da Faculdade de Medicina de Ribeirão Preto da Universidade de São Paulo

I Pós-graduando do Departamento de Ginecologia e Obstetrícia da Faculdade de Medicina de Ribeirão Preto da Universidade de São Paulo - USP - Ribeirão Preto (SP), Brasil.

2 Professor Associado do Departamento de Ginecologia e Obstetrícia da Faculdade de Medicina de Ribeirão Preto da Universidade de São Paulo - USP - Ribeirão Preto (SP), Brasil.

${ }_{3}^{3}$ Professor Associado do Departamento de Bioquímica e Imunologia da Faculdade de Medicina de Ribeirão Preto da Universidade de São Paulo - USP - Ribeirão Preto (SP), Brasil.

${ }^{4}$ Doutor, Professor do Departamento de Ginecologia e Obstetrícia da Faculdade de Medicina de Ribeirão Preto da Universidade de São Paulo - USP - Ribeirão Preto (SP), Brasil.

5 Professor Titular do Departamento de Ginecologia e Obstetrícia da Faculdade de Medicina de Ribeirão Preto da Universidade de São Paulo - USP - Ribeirão Preto (SP), Brasil.

Não houve auxílio sob forma de financiamento, equipamentos ou fornecimento de drogas.

Aceito com modificacões 


\section{Introdução}

A correção cirúrgica da agenesia vaginal está indicada se não houver possibilidade de se obter neovagina por técnicas conservadoras ${ }^{1,2}$ ou houver intenção de uni-la a um útero funcionante existente. A intenção, com isso é permitir a ocorrência de coitos e, em casos individualizados, de menstruações e gestação espontânea.

A neovaginoplastia foi descrita por McIndoe e Bannister ${ }^{3}$ com o revestimento da cavidade neovaginal com enxerto de pele abdominal. Entretanto, as implicações estéticas sobre o sítio doador e a incidência elevada de necrose e infecções ${ }^{4,5}$ estimularam inúmeros autores a desenvolver alternativas para a enxertia como modificação à técnica, sempre com intuito de reduzir a morbidade intra- e pós-operatória.

Ao longo das últimas duas décadas, utilizamos, em nosso serviço, o aloenxerto neovaginal de âmnio humano, com resultados animadores quanto à ocorrência de coitos e à satisfação das pacientes ${ }^{5-8}$. Entretanto, pela possibilidade da transmissão de infecções e rejeição, comum a todos os procedimentos que envolvem enxerto de doador, temos aplicado nova modificação à técnica, com o uso de moldes de látex natural (Hevea brasiliensis), sem o revestimento de enxerto biológico. Seu uso como material biosintético encontra respaldo no bom desempenho observado na cicatrização de úlceras cutâneas ${ }^{9}$, como prótese pericárdica ${ }^{10}$, e, mais recentemente, na correção de defeitos timpânicos ${ }^{11} \mathrm{e}$ na incorporação de implantes dentários ${ }^{12}$.

O objetivo deste estudo piloto foi avaliar os resultados obtidos a partir do uso de molde de látex natural como modificação à neovaginoplastia de McIndoe e Bannister, contemplando aspectos morfológicos (epitelização e vaginometria) e funcionais (ocorrência de coitos e satisfação da paciente) da neovagina, bem como complicações associadas ao procedimento.

\section{Métodos}

Para esta avaliação preliminar, foram revisados os prontuários de nove pacientes com diagnóstico de síndrome de Mayer-Rokitansky-Küster-Hauser (MRKH), submetidas à neovaginoplastia pela técnica de McIndoe e Bannister ${ }^{3}$, modificada pela utilização do molde de látex natural. Estas pacientes foram atendidas no Ambulatório de Ginecologia Infanto-puberal do Hospital das Clínicas da Faculdade de Medicina de Ribeirão Preto da Universidade de São Paulo (FMRP-USP), entre os anos de 2002 e 2006. A idade das pacientes ao diagnóstico variou de 15 a 31 anos (mediana $=16$ ) e ao tratamento, de 15 a 38 (mediana $=19$ ). Duas pacientes possuíam útero hipoplásico não funcionante e as demais, agenesia de útero e vagina. Como malformações associadas, agenesia renal unilateral foi observada em dois casos e uma paciente apresentava estenose de esôfago e malformações da orelha.

Todas as pacientes receberam profilaxia antibiótica pré-operatória com 2,0 g de cefazolina, pela via intravenosa, no momento da indução anestésica. A confecção cirúrgica da neovagina iniciou-se pela identificação da depressão em fundo cego, que, em pacientes normais, corresponderia ao intróito vaginal (Figura 1A), sondagem uretral e abertura transversal da mucosa (Figura 1B). Seguiu-se a dissecção romba para obtenção de espaço entre a bexiga e o reto, com divulsão digital do tecido conjuntivo frouxo, confeccionando o túnel neovaginal com movimentos laterais (Figura $1 \mathrm{C}$ ) até uma profundidade aproximada de $8,0 \mathrm{~cm}$.
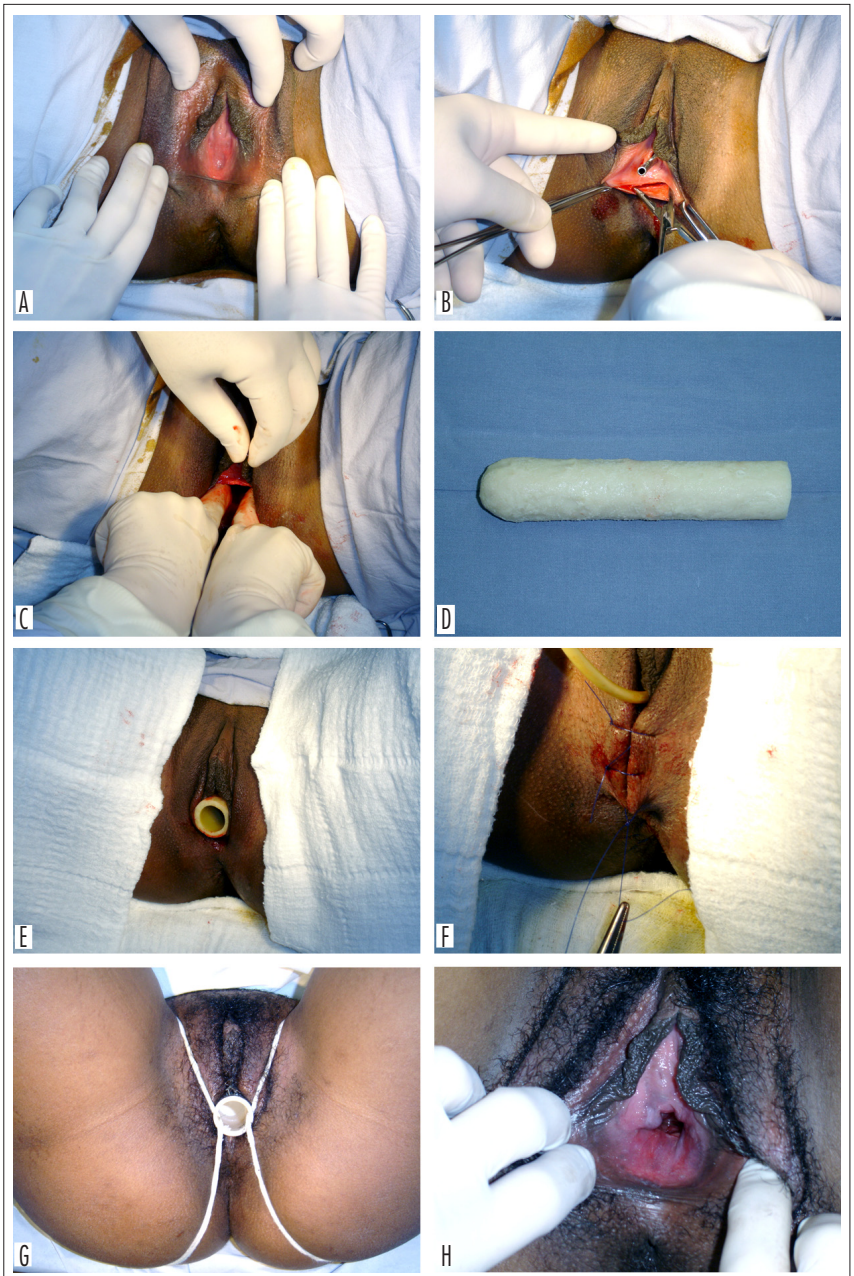

Figura 1 - (A) Exposição do vestíbulo vaginal, evidenciando depressão em fundo cego, que corresponderia ao intróito; (B) abertura transversal da mucosa, identificando o espaço vésico-retal, após sondagem para esvaziamento vesical e identificação do trajeto uretral; (C) divulsão digital látero-tateral do tecido conjuntivo frouxo, para formar o túnel neovaginal, com profundidade aproximada de $8,0 \mathrm{~cm}$; (D) molde de látex natural; (E) posicionamento do molde sepultado na cavidade obtida; (F) fixação do molde no interior da neovagina, por pontos separados de fio não absorvível aproximando os lábios maiores; (G) fixação do molde no pós-operatório tardio, por cadarços ajustados a uma cinta de algodão; $(H)$ epitelização da neovagina após 40 dias do procedimento. 
Obtido túnel adequado e revista a hemostasia, o molde de látex natural (Figura 1D) foi sepultado na cavidade neoformada (Figura 1E) e fixado pela aproximação dos lábios maiores (Figura 1F), mantendo-o firme, porém sem muita tensão. Os diâmetros do molde utilizado variaram entre 2,5 e $3,5 \mathrm{~cm}$, de acordo com as características fenotípicas de cada paciente.

Após alta hospitalar precoce, o molde vaginal permaneceu na neovagina até o primeiro retorno, sete dias depois do procedimento. Uma vez retirado o molde e realizada a higiene da neovagina com solução fisiológica sob pressão, realizou-se a primeira avaliação da epitelização da neovagina e procedeu-se à colocação de um novo molde de látex natural, cuja fixação foi feita por uma cinta de algodão adequada à cintura da paciente, por cadarços justos o suficiente para manter o molde no interior do túnel neovaginal (Figura 1G), sem grande desconforto.

Até o retorno seguinte, as pacientes foram encorajadas a retirar o molde diariamente durante o banho, reposicionando-o em seguida, depois de untado com creme vaginal com antimicrobiano de amplo espectro para lubrificação. O segundo retorno foi agendado após 40 dias do procedimento, com objetivo de avaliar a cavidade neovaginal quanto à epitelização, amplitude do intróito e o comprimento do túnel pela vaginometria. Constatada a evolução favorável, liberamos as pacientes para atividade sexual, que, se mantida regularmente, dispensaria o uso do molde. Sempre que houvesse períodos prolongados de abstinência sexual, as pacientes deveriam fazer uso regular do molde durante o sono, para manutenção da cavidade funcional.

Foram analisadas as anotações em prontuário, referentes à evolução da epitelização e da vaginometria, às complicações precoces e tardias, à ocorrência de coitos e à satisfação das pacientes, a partir da queixa de dispareunia ou outras disfunções.

\section{Resultados}

A duração do procedimento cirúrgico foi inferior a 60 minutos e o sangramento intra-operatório foi considerado pequeno em todos os casos operados. A dor pós-operatória foi considerada de baixa intensidade por todas as pacientes. Oito pacientes receberam alta precoce, 24 horas após o procedimento, e uma internação foi prolongada para tratamento clínico de pielonefrite não complicada.

No oitavo dia pós-operatório, todas as pacientes apresentavam cavidade neovaginal recoberta por fino epitélio em formação, sem sinais de necrose, infecção secundária ou outras complicações.

No retorno, após cinco semanas da cirurgia, oito pacientes apresentavam neovagina adequadamente epitelizada
(Figura $1 \mathrm{H}$ ) e de profundidade satisfatória, variando de 7 a $12 \mathrm{~cm}$ (média de $8,8 \mathrm{~cm}$ ). Uma paciente deste grupo (Paciente 2) apresentava estenose leve do terço distal da neovagina, tendo sido orientada a fazer exercícios de pressão com o molde.

Como complicações precoces, foram observadas, ainda, uma fístula retovaginal (Paciente 4), com pronta correção cirúrgica, e uma estenose completa da neovagina (Paciente 5), atribuída ao abandono do molde no $12^{\circ}$ dia pós-operatório.

Todas as oito pacientes com neovagina considerada satisfatória foram liberadas e iniciaram coitos após seis semanas. Destas, em vista de dificuldades geográficas e sociais, duas perderam seguimento após quatro meses; mas, até a última consulta, ambas afirmavam coitos regulares e satisfatórios, sem qualquer disfunção. Para as demais pacientes, em um seguimento mínimo de um ano, observamos a manutenção de neovaginas de profundidade satisfatória, variando de 4 a $8 \mathrm{~cm}$ (média de $6,7 \mathrm{~cm}$ ), e a capacidade para o coito.

Três pacientes apresentaram complicações tardias. Houve um caso de encurtamento da neovagina no $55^{\circ}$ dia pós-operatório (Paciente 8), para a qual também foram recomendados os exercícios de pressão com molde vaginal rígido, tendo evoluído com vaginometria de $7 \mathrm{~cm}$ após nove meses de acompanhamento. Esta paciente, após seguimento de um ano, referia coitos regulares, porém disfuncionais, com dispareunia profunda e anorgasmia. A Paciente 2, que havia apresentado estenose do terço distal da neovagina, evoluiu com estenose do terço médio e, não respondendo aos exercícios de pressão, foi submetida a nova neovaginoplastia com molde de látex natural, um ano após o primeiro procedimento. Evoluiu satisfatoriamente, constatando-se atualmente neovagina com $4 \mathrm{~cm}$ de profundidade, permissiva aos coitos, que são regulares e satisfatórios, sem qualquer disfunção. Por fim, a Paciente 4, que apresentou fístula retovaginal precoce, evoluiu com fístulas uretrovaginais recidivantes, tendo sido necessárias sucessivas correções cirúrgicas e uma nova neovaginoplastia com molde de látex natural, em um ano e meio de seguimento. Três anos após a primeira cirurgia, esta paciente referia perdas urinárias aos coitos e dispareunia superficial, com vaginometria de $7 \mathrm{~cm}$.

Observamos taxa de satisfação em relação aos coitos de $75 \%$ numa avaliação precoce e de $66,7 \%$ na avaliação após um ano da cirurgia. Ocorreram complicações em quatro pacientes $(44,4 \%)$, com resolução completa em um caso (coitos funcionais) e dois casos com resolução parcial (permaneceram coitos disfuncionais), sendo apenas um caso de complicação não resolvida, por estenose completa da neovagina e abandono do seguimento ambulatorial (Tabela 1). 
Tabela 1 - Informações sobre o seguimento das pacientes submetidas a neovaginoplastia com molde de látex natural.

\begin{tabular}{|c|c|c|c|c|}
\hline Paciente & $\begin{array}{c}\text { Tempo de seguimento } \\
\text { (meses) }\end{array}$ & $\begin{array}{l}\text { Vaginometria } \\
(\mathrm{cm})\end{array}$ & Coitos & $\begin{array}{l}\text { Sutisfaccão/ } \\
\text { disfunção coitos }\end{array}$ \\
\hline 1 & 4 & 5 & Sim & Satisfatório \\
\hline 2 & 48 & 4 & Sim & Satisfatório \\
\hline 3 & 36 & 8 & Sim & Satisfatório \\
\hline 4 & 36 & 7 & Sim & $\begin{array}{l}\text { Perdas urinárias e } \\
\text { dispareunia superficial }\end{array}$ \\
\hline 5 & 1 & 0,5 & Não & \\
\hline 6 & 24 & 6 & Sim & Satisfatório \\
\hline 7 & 4 & 7 & Sim & Satisfatório \\
\hline 8 & 12 & 7 & Sim & $\begin{array}{l}\text { Dispareunia profunda e } \\
\text { anorgasmia }\end{array}$ \\
\hline 9 & 12 & 8 & Sim & Satisfatório \\
\hline
\end{tabular}

\section{Discussão}

O objetivo de se realizar neovaginoplastia em pacientes com a síndrome de MRKH é proporcionar a ocorrência de coitos, em que pesem não apenas a penetração, mas, principalmente, o conforto e a satisfação da paciente. Tendo em vista as dificuldades de adaptação e o impacto psicológico causado pelo problema em si, além daquelas características, a técnica aplicada deve oferecer recuperação pós-operatória livre de complicações, sejam elas decorrentes do ato cirúrgico em si ou da morbidade causada por seqüelas estéticas e funcionais.

O seguimento ambulatorial das pacientes submetidas à neovaginoplastia deve ter reavaliações próximas nos primeiros meses, com o intuito de acompanhar a evolução morfológica e funcional da neovagina, bem como a freqüência de coitos e/ou a execução correta dos exercícios com o molde. A importância da disciplina na administração pós-operatória do molde confirma-se pela ocorrência de estenose e encurtamento do túnel neoformado nos primeiros dois meses após o procedimento em pacientes que referiram não tê-lo utilizado adequadamente. Esse tipo de complicação já foi descrito em pacientes submetidas à técnica de Abbé-McIndoe com enxerto cutâneo glúteo ${ }^{13}$. Em nossa casuística, como a vontade de iniciar atividade sexual foi critério para a indicação cirúrgica, não constatamos perda da neovagina por ausência de coitos.

Observamos que algumas pacientes buscam a correção cirúrgica para a agenesia vaginal antes da maioridade, marcando a realidade da iniciação sexual cada vez mais precoce em nosso país ${ }^{14}$. A constatação nos faz atentar para a necessidade de se garantir o acompanhamento pós-operatório ainda mais cuidadoso, uma vez que, na adolescência, as condições psicológicas e as mudanças de comportamento podem ser determinantes do bom resultado cirúrgico, que depende da adesão da paciente às recomendações de uso do molde.

Conquanto as principais adversidades derivem da baixa adesão ao uso do molde vaginal, podem existir outras complicações, referentes ao ato cirúrgico em si, com destaque para as fístulas, que podem envolver reto, bexiga e uretra. Tais complicações são descritas também para as demais técnicas existentes ${ }^{15,16}$, mas, em absoluto, contra-indicam qualquer uma delas, uma vez que a correção cirúrgica é de fácil execução por equipes experientes e, em geral, não estão associadas a estenose da neovagina.

Em nosso serviço, a avaliação morfológica do resultado cirúrgico foi considerada satisfatória, considerando a amplitude permissiva ao toque vaginal bidigital, a vaginometria média de $8,8 \mathrm{~cm}$, em seguimento mínimo de um ano, e a epitelização precoce da neomucosa, observada em todas as pacientes. Os resultados confirmaram as propriedades do látex natural derivado de Hevea brasiliensis, de reposição e regeneração tecidual e de aceleração do processo de cicatrização?. À revelia da morbidade intra e pós-operatória potencialmente elevada, outras técnicas permitem a obtenção de neovaginas com características semelhantes ${ }^{5,13,17-19}$.

A avaliação do resultado cirúrgico, contudo, não deve contemplar apenas o aspecto e as medidas da neovagina ou a ocorrência de coitos regulares, mas também a satisfação da mulher com o coito, fundamental para o exercício pleno de sua sexualidade. Keser et al. ${ }^{13}$, em 2005 , avaliaram a função das neovaginas apenas a partir de relatos dos parceiros, o que demonstra claramente a persistência de uma noção antiquada de que o ato sexual se resume à seqüência penetração-ejaculação.

Para avaliação funcional, consideramos satisfatórios os coitos em que havia penetração plena e prazerosa, sem qualquer queixa de dor ou desconforto. Conduzido o pós-operatório adequadamente, obtivemos um índice de satisfação de $75 \%$ nos primeiros meses e de $66,7 \%$ após o tempo mínimo de um ano da cirurgia. Observamos ser a dispareunia a complicação mais freqüente no início da atividade sexual, assim como ocorre com desejo sexual hipoativo e anorgasmia. Esta observação é provável resultado de um período de adaptação à neovagina, marcado por alguma insegurança em relação à capacidade para o ato sexual pleno para ambas as partes. Ainda acreditamos que o grande incômodo no período pós-operatório possa gerar alguma resistência da paciente em aceitar a neovagina como um órgão funcional, o que justifica e exige o oferecimento de consultas direcionadas à sexualidade desde o período préoperatório, como estratégia de profilaxia do problema.

Concluindo, além do fácil manuseio, dos tempos reduzidos de cirurgia e internação e da baixa incidência de dor pós-operatória, o uso do molde de látex natural (Hevea brasiliensis) como modificação à técnica de $\mathrm{McIndoe} \mathrm{e} \mathrm{Bannister}{ }^{3}$ proporcionou às pacientes com síndrome de MRKH a obtenção de neovaginas de aspecto muito semelhante à vagina normal e a ocorrência de coitos satisfatórios. Por isso, surge como uma boa opção cirúrgica frente às demais técnicas vigentes, trazendo como vantagem a baixa morbidade e a ausência dos riscos associados ao uso de auto e aloenxertos. 


\section{Referências}

1. Frank RT. The formation of an artificial vagina without operation. Am J Obstet Gynecol. 1938;35:1053-5.

2. Ingram JM. The bicycle seat stool in the treatment of vaginal agenesis and stenosis: a preliminary report. Am J Obstet Gynecol. $1981 ; 140(8): 867-73$.

3. Mclndoe $\mathrm{AH}$, Bannister JB. An operation for the cure of congenital absence of the vagina. J Obstet Gynaecol Br Emp. 1938:45:490-4

4. Sá MFS, Baruffi I, Cardoso AA. Agenesia de vagina: correção cirúrgica pela técnica de Mclndoe. Rev Bras Ginecol Obstet. 1986;8(5):156-9.

5. Moura MD, Ferriani RA, Sá MFS, Wanderley MS, Leite SP, Soares FA. Epitelização vaginal com membrana amniótica em neovaginoplastia. Rev Bras Ginecol Obstet. 1994;16(3/4):135-40.

6. Moura MD, Sá MFS, Ferriani RA, Sala MM, Soares F. Epitelización vaginal con membrana amniótica en neovaginoplastia. Obstet Ginecol Esp. 1991;1(5):193-7.

7. Ferriani RA, Carvalho RM, Moura MD, Sá MFS. Neovaginoplastia: comparação entre técnicas cirúrgicas. Rev Bras Ginecol Obstet. 1996; 18(4):343-7.

8. Moura MD, Navarro PA, Nogueira AA. Pregnancy and term delivery after neovaginoplasty in a patient with vaginal agenesis. Int J Gynaecol Obstet. 2000;71 (3):215-6.

9. Mrué F, Coutinho Netto J, Ceneviva R, Lachat JJ, Thomazini $\mathrm{JA}$, Tambelini $\mathrm{H}$. Evaluation of the biocompatibility of a new biomembrane. Mat Res. 2004;7(2):277-83.

10. Sader SL, Coutinho Netto J, Barbieri Neto J, Mazzetto SA, Alves Júnior $P$, Vanni JC, et al. Substituição parcial do pericárdio de cães por membrana de látex natural. Rev Bras Cir Cardiovasc. 2000;15(4):338-44.
11. Oliveira JAA, Hyppolito MA, Coutinho Netto J, Mrué F. Miringoplastia com a utilização de um novo material biossintético. Rev Bras Otorrinolaringol. 2003;69(5):649-55.

12. Balabanian $C A$, Coutinho Netto J, Lamano-Carvalho TL, Lacerda SA, Brentegani LG. Biocompatibility of natural latex implanted into dental alveolus of ratos. J Oral Sci. 2006;48(4):201-5.

13. Keser A, Bozkurt N, Taner ÖF, Sensöz Ö. Treatment of vaginal agenesis with modified Abbé-Mclndoe technique: long-term follow-up in 22 patients. Eur J Obstet Gynecol Reprod Biol. $2005 ; 121(1): 110-6$.

14. Abramovay M, Castro MG, Silva LB. Juventude e sexualidade. Brasília: Unesco; 2004.

15. Alessandrescu D, Peltecu GC, Buhimschi CS, Buhimschi IA. Neocolpoiesis with split-thickness skin graft as a surgical treatment of vaginal agenesis: retrospective review of 201 cases. Am J Obstet Gynecol. 1996;175(1):131-8.

16. Mizia K, Bennett M, Dudley J, Morrisey J. Müllerian dysgenesis: a review of recent outcomes at Royal Hospital for Women. Aust N Z J Obstet Gynaecol. 2006;46(1):29-31.

17. 1: Motoyama S, Laoag-Fernandez JB, Mochizuki S, Yamabe S, Maruo T. Vaginoplasty with Interceed absorbable adhesion barrier for complete squamous epithelialization in vaginal agenesis. Am J Obstet Gynecol. 2003;188(5):1260-4.

18. Lin WC, Chang CY, Shen YY, Tsai HD. Use of autologous buccal mucosa for vaginoplasty: a study of eight cases. Hum Reprod. 2003;18(3):604-7.

19. Sharma JB, Gupta N, Mittal S. Creation of neovagina using oxidized cellulose (surgicel) as a surgical treatment of vaginal agenesis. Arch Gynecol Obstet. 2007;275(4):231-5. 